\title{
The Abuse of Animals and Domestic Violence: A National Survey of Shelters for Women who are Battered
}

\section{Frank R. Ascione, Claudia V. Weber, and David S. Wood ${ }^{1}$ UTAH STATE UNIVERSITY}

The maltreatment of animals, usually companion animals, may occur in homes where there is domestic violence, yet we have limited information about the prevalence of such maltreatment. We surveyed the largest shelters for women who are battered in 49 states and the District of Columbia. Shelters were selected if they provided overnight facilities and programs or services for children. Ninety-six percent of the shelters responded. Analysis revealed that it is common for shelters to serve women and children who talk about companion animal abuse. However, only a minority of respondents indicated that they systematically ask about companion animal maltreatment in their intake interview. We discuss the implications of these results for domestic violence programs, animal welfare organizations, and programs serving children of women who are battered by their partners.

"A moment later, Francine heard Nicky scream...'Nicky was crying so hard she couldn't talk. I'd never heard a child cry like that. I...held her in my arms until she calmed down enough to tell me what had happened. Mickey (Francine's husband) had warned her that if he found the cat on the porch he'd wring its neck. When he caught her with it the second time he took it out of her arms and just broke its neck in his two hands" (McNulty, 1989, p. 165).

"Francine Hughes was charged with the death by fire of her husband, Mickey Hughes, in 1977”' (McNulty, 1989, author's note).

"[Her lawyer] asked Francine to tell the story of their pet dog, Lady. As Francine described Lady's death (Mickey had refused to allow his family to assist Lady while she was giving birth) a shockwave of emotion swept the courtroom. The simplicity of the event - a helpless animal, a female, 
left outside to freeze while struggling to give birth — held no ambiguity, no shadings of motive; it left no room for doubt. The impact of the story was as strong as anything Francine had told so far" (McNulty, 1989, p. 258).

Although an age-old issue, the relationship between the abuse and maltreatment of nonhuman animals and human interpersonal violence is receiving renewed attention from the scientific community. Two recent reviews of literature (Arkow, 1996; Ascione, 1993) highlight the potential confluence of child maltreatment, domestic violence, and animal maltreatment. Each form of abuse can occur independently or in combination with other forms of violence.

The present study is the outgrowth of a series of projects specifically examining the dynamics of human-perpetrated violence toward animals (herein used to refer to nonhuman animals). Following a brief overview of related literature, we report the results of a national survey of shelters for women who are battered. This survey was conducted to evaluate shelters' experience with reported animal maltreatment and to find out whether shelters systematically assess this form of abuse. We conclude with discussion of policy and therapeutic issues that need to be addressed as animal welfare organizations and domestic violence programs embark on more collaborative efforts to deal with violence toward animals and people.

\section{Overview}

An earlier paper (Ascione, 1993) outlined a series of issues that pertain to the development of cruelty toward animals in childhood and adolescence, using the following definition of cruelty: "...socially unacceptable behavior that intentionally causes unnecessary pain, suffering, or distress to and/or death of an animal..." (p. 228). Case examples from the early psychoanalytic literature were reviewed as well as primarily retrospective research from forensic psychiatry and sociology linking childhood histories of animal abuse with contemporary patterns of criminal violence. One of the watershed events for research in this area was the inclusion of "cruelty to animals" among the symptoms of Conduct Disorder in children and adolescents in major psychiatric diagnostic manuals (American Psychiatric Association, 1987, 1994). Conduct Disorder represents a pattern of antisocial behavior that can persist into adulthood.

Research examples included the association of animal maltreatment with cases of child physical abuse, the sexual abuse of children, and partner battering or 
domestic violence. Follow-up work by collcagues and the present authors has included the design and field testing of a questionnaire for assessing children and adolescents' histories of animal abuse (Ascione, Thompson, \& Black, in press) and a survey on animal maltreatment for use with women who have been battered (Ascione, in press; Ascione \& Weber, 1995).

Since this review, there have been a number of publications attempting to raise the consciousness of the child welfare community (Boat, 1995) and the veterinary profession (Arkow, 1994; Munro, 1996) about the need to attend to the maltreatment of animals. However, similar advances are only beginning within the community of professionals who deal with domestic violence.

\section{Animal Abuse and Domestic Violence}

Much of the information we have about the relationship between the maltreatment of animals and partner battering is derived from anecdotal reports (like the passages quoted at the opening of this paper) in the literature on domestic violence. Hogarth (1750/1751; Shesgreen, 1973, plates 77-79) provided an artistic representation of the relation in his series of engravings entitled, "The Stages of Cruelty" in which childhood cruelty to animals progressed to a fatal domestic assault. In 1809, the psychiatrist Pinel provided a similar example from his case histories (cited in Berrios, 1996).

Anecdotally, we also know that animals have been abused by perpetrators to frighten their partners, as a threat of potential interpersonal attacks, and as a form of retaliation or punishment, and that abuse has been implicated in forced bestiality. That children are often witness to such displays of cruelty has received scant research scrutiny.

\section{Child Witnesses to Domestic Violence and Animal Abuse}

“...[T] he Custom of [children] Tormenting and Killing ...Beasts, will, by Degrees, harden their Minds even towards Men; and they who delight in the Suffering and Destruction of inferiour Creatures, will not be apt to be very compassionate, or benign to those of their own kind." (Locke, 1705, section 116, cited in Axtell, 1968).

Recently, Jouriles, Norwood, McDonald, Vincent, and Mahoney (1996) reported on the results of a study of two samples in which domestic violence was present, one including couples seeking marital therapy and the other women seeking shelter 
from battering. They found that physical violence and other forms of marital aggression were associated with externalizing (acting out) problems in the 5 to 12 year-old children in these families. It is important to note that cruelty to animals is often included as a component of externalizing problems.

This is one example of the increased research attention being paid to the effects of witnessing family violence on the psychological adjustment of children, a group sometimes referred to as the "forgotten victims" of partner abuse (Jaffe \& Sudermann, 1995; Osofsky, 1995). However, there has been virtually no research on circumstances in which children may witness not only the battering of their parent (most often the mother) but also the abuse of their beloved companion animals, a combination that may compound these children's trauma and contribute to their psychological maltreatment. It should be noted that children in such homes are at heightened risk for being abused themselves. Witnessing parent and companion animal abuse may compromise children's psychological adjustment, increase their propensity for interpersonal violence (via observational learning and/or identification with the aggressor), and make children's cruelty to animals more likely to emerge as a symptom of their distress.

\section{Women who Seek Shelter from Battering}

To our knowledge, there are only three reports, two of which are unpublished, on the experience of women in battered women's shelters with threatened and actual abuse of animals. Arkow (1996) cited two studies, one of which was conducted at the Center for Prevention of Domestic Violence in Colorado Springs, Colorado and found that $24 \%$ of women $(N=122)$ seeking safehouse refuge reported that their abusers had abused animals in their presence. The other study was conducted by the La Crosse, Wisconsin Community Coalition against Violence among 72 women using domestic violence prevention services. Eighty-six percent of these women reported having companion animals and, of these women, $80 \%$ had experienced their partners' maltreatment of companion animals.

Ascione (in press), in collaboration with a shelter in northern Utah for women who are battered, surveyed 38 women entering the shelter for in-house services. Using a form of the Battered Partner Shelter Survey (BPSS) - Pet Maltreatment Assessment (Ascione \& Weber, 1995), he found that $74 \%$ of the women reported having a companion animal currently or in the past twelve months. Of these women, $71 \%$ indicated that their boyfriend or husband had either threatened harm to their animals or had engaged in actual maltreatment and/or killing of an animal. The prevalence of companion animal abuse by children in these families was also 
disturbingly common. Thirty-two percent of the 22 women with children gave examples of children hurting or killing animals. This level of cruelty is comparable to what has been found in samples of mental health clinic child clients (Achenbach \& Edelbrock, 1981; Achenbach, Howell, Quay, \& Conners, 1991) and in a sample of sexually abused children (William Friedrich, April, 1992, personal communication). In this sample of women with companion animals, nearly one in five (18\%) reported that they had delayed entering the shelter because of concerns about their companion animal's safety.

Given the small and regionally narrow sample of women recruited for these studies, we thought it would be valuable to derive some sense of the national scope of this problem. As I note in a forthcoming article (Ascione, in press):

Although this study [of the 38 shelter women] did not include comparison samples of non-battered women or women who are not currently in shelters, the substantial rate of partner cruelty to animals is clearly a cause for concern. Caution must be exercised in generalizing from this study's sample to state and national samples; however, extrapolation of this study's findings may help estimate the scope of the potential problem. For example, two million is a conservative estimate of the number of U.S. women assaulted by their male partners each year (see Browne, 1993). If half of these women have companion animals (again, a conservative estimate [Ascione, 1992]), 71\% partner cruelty to animals represents hundreds of thousands of families where companion animal victimization, actual or threatened, is part of the landscape of terror to which some women are exposed.

Since systematic data collection about the prevalence of cruelty to animals reported by women who enter safehouses or shelters is uncommon at the state level, we decided to conduct a national survey, selecting one shelter per state and the District of Columbia. We excluded Utah shelters from our sampling because of an ongoing study that specifically addresses cruelty to animals (Ascione, Weber, Thompson, \& Wood, in preparation). Our purposes were to survey shelter personnel about their perceptions regarding the overlap between domestic violence and animal maltreatment, ascertain whether women and children coming to shelters mention companion animal abuse, and find out whether shelters routinely collect information about the abuse of companion animals in their intake protocol. If shelters collected such information, we asked about the type of information gathered. 


\section{Method}

\section{Sampling Procedure}

We obtained the most recent edition (1994) of the National Directory of Domestic Violence Programs compiled by the National Coalition against Domestic Violence (NCADV). The directory contains state-by-state information as well as information from Washington, D.C., Puerto Rico, and the U.S. Virgin Islands, derived from a survey conducted by the Coalition. The number of programs listed for each state ranged from 4 (Delaware) to 120 (New York). We elected not to include Puerto Rico and the Virgin Islands in our sampling.

The directory lists 16 potential service categories for each program, three of which were relevant for the current study. These were: "SHELTER - Residential facility for battered women and their children...CHILDREN'S COUNSELING/ PROGRAMS - One or more of the various services provided for children of battered women ranging from counseling, to advocacy, recreational activities, and a structured children's program...SHELTER CAPACITY - The number of women and children who can be sheltered at any one time" (NCADV, 1994, unpaginated).

We selected one shelter from each state (excluding Utah) and the District of Columbia using the following criteria: the facility had to provide overnight (residential) accommodations, indicate that children's counseling or programs were available, and be the largest shelter in the state based on "shelter capacity" as defined above. In most cases, we selected the shelter located in a major, well-known city.

\section{Instrument}

We developed a simple, one-page questionnaire with seven items and space for open-ended comments, since we were aware of the premium on time for most shelter personnel. The exact wording of each item will be provided in the Results section but, in brief, the questionnaire asked about the number of clients served in a six-month period, whether women or children coming to the shelter mentioned companion animal abuse, and whether the respondents had noted the coexistence of domestic violence and companion animal abuse and, if so, their estimate of the extent of overlap between these forms of violence. We also asked whether a question about companion animal abuse was included in the shelter's intake interview. 
A cover letter describing the project as a study of the "relation between domestic violence toward women and children and abuse of companion animals" was attached to the questionnaire together with a stamped, addressed return envelope. The study protocol, letter, and questionnaire were approved by Utah State University's Institutional Review Board (IRB) for Human Subjects Research, and a copy of the IRB approval was included in the mailing.

\section{Survey Procedure}

After an initial mailing, a second mailing was made to sites that had not responded. Following two mailings, shelters were contacted by phone and the questionnaire was administered as an interview (for those sites that agreed to participate). Respondents primarily were shelter directors and front line staff having direct contact with clients.

\section{Results}

Our survey of 50 shelter programs yielded responses from 48, representing a $96 \%$ response rate. One program did not respond despite repeated phone calls and one declined participation due to time constraints.

We asked shelters to indicate the number of women who stayed in their facility at least one night during the period November 1, 1995 to May 1, 1996. Estimates were provided by $87.5 \%$ of the shelters, with $12.5 \%$ either unable to provide an estimate or leaving this item blank. For the 42 shelters completing this item, the number of women staying overnight during the six-month period ranged from 34 to 600 , with a mean of 186 .

One of the questions we asked (see Table 1 for a summary of results) was, "Do women who come in to your shelter talk about incidents of pet abuse?" An affirmative response was given by $85.4 \%$ of the shelters. In response to the question, "Do children who come in to your shelter talk about incidents of pet abuse?" 63\% of the 46 shelters that completed this item said "Yes."

We also asked respondents, "In your experience with shelters, have you observed the coexistence of domestic violence and pet abuse?" Eighty-three point three percent of the shelters responded affirmatively. When asked, "What is your best estimate of the percentage of homes where domestic violence and pet abuse coexist?" Fifty percent of the shelters provided estimates (the remaining shelters either entered a question mark or left this item blank). Estimates ranged from less 


\section{Table 1. Percent Respondents Answering "Yes" to Each Question}

\section{Questions}

"Yes" (\%)

Do women who come into your shelter talk about incidents of companion animal abuse?

Do children who come into your shelter talk about incidents of companion animal abuse?

In your experience with shelters, have you observed the coexistence of domestic violence and companion animal abuse?

Do you have any questions in your intake interview concerning companion animals?

than $1 \%$ to $85 \%$ with a mean estimate of $44 \%$ coexistence of domestic violence and companion animal abuse.

In response to the question, "Do you have any questions in your intake interview concerning pets?", 27.1\% responded "Yes." (Estimates of percent coexistence were unrelated to whether shelters had a question about companion animals in their intake interview.) Of the 13 shelters responding affirmatively, 12 responded to a follow-up question about the type of questions asked during intakes. A sampling of responses included:

- "Has he threatened to hurt you, your family, your pets or favorite belongings of yours?"

- "Has abuser threatened to harm or ever harmed pet in the household?"

- 'In a section called 'History of Abuse,' we ask if they've experienced pet abuse."

- "Do you have animals at home? Are they safe?"

- "Has there been physical destruction of property/pets? Where are the companion animals? Do you have a safe place to keep them?"

Finally, we asked respondents if they wished to receive a brief summary of the study when it was completed. This item was left blank by $46 \%, 42 \%$ requested a copy, and $12 \%$ said "No." 


\section{Discussion}

Before discussing the results of this study, a few caveats are in order. There are hundreds of domestic violence programs throughout the United States - we sampled only 50 of these which met our selection criteria. Therefore, caution should be exercised in generalizing our results to all shelters. Since we selected the shelter with the largest capacity in each state, we probably excluded shelters serving primarily rural communities where farm and wild animal abuse may occur, another factor limiting generalization. Finally, a survey of nonresidential domestic violence programs and shelters that do not provide children's services would be valuable as a comparison for the data we obtained.

The overwhelming majority of shelters we surveyed indicated that women seeking shelter mention experiences of companion animal abuse. A smaller but still substantial majority also reported that children have shared instances when companion animals have been abused in their homes. In fact, shelters reporting that children talked about companion animal abuse always reported that women discussed companion animal abuse as well. Despite the fact that 40 of the 48 shelters believed that domestic violence and companion animal abuse coexist, only 13 shelters specifically assess this issue in their intake interview. Some factors that may account for the discrepancy between awareness of the link between animal maltreatment and domestic violence and the apparent failure to explore the link with shelter clients include the limited time that shelter staff can devote to intake processes and uncertainty about how to deal with animal welfare issues that might arise. In the remainder of this discussion, we explore issues that may be valuable to consider as collaborative programs between domestic violence services and animal welfare organizations continue to evolve.

\section{Implications for Domestic Violence Programs}

We have not given sufficient attention to cruelty to animals as an indicator of partner dangerousness or lethality. A brief question or two about animal maltreatment in intake interviews and crisis call interview protocols could provide information about a partner's capacity for physical violence. We also must be alert to animal cruelty as an indication that violence may be escalating. Although it has not been explored systematically, this information could also enhance prosecution efforts by helping establish patterns of physical violence.

We know that there may be repeated visits to shelters and numerous crisis calls before a woman leaves a batterer. If companion animals are present in these cases, 
incorporating information about animal welfare in safety planning for women who continue to live with their batterer is essential. Concern for companion animal welfare may actually delay a woman's seeking of shelter, and this is an obstacle that could be removed. Domestic violence victim advocates who arrive at a scene after police have "secured" the location should also be trained to ask about companion animal welfare to assist women in their decisions about remaining at home or seeking shelter. Information about a batterer's history of animal abuse could also be considered in requesting protective orders.

As of February 1997, the Utah legislature is considering a bill that would increase penalties in domestic violence cases where children witness family violence. It might be advisable to include witnessing the abuse and killing of companion animals as a further traumatizing experience for children in whose homes partner battering occurs.

One final consideration is our need for a better understanding of the dynamics of animal abuse in families where there is domestic violence. We know that cruelty to animals may be a battering partner's attempt at control, coercion, intimidation, retaliation, and an element of forced bestiality. However, we know little about battering victims' reactions to and interpretations of such events. If a women has experienced animal maltreatment by her partner, under what circumstances does this further immobilize her, heightening her fear of leaving (especially when weapons have been used) and when does it prompt her to escape an abusive situation?

\section{Implications for Animal Welfare Organizations}

In many instances, animal welfare organizations have taken the lead in promoting collaborative programs to reduce violence to all vulnerable victims, human and nonhuman. Yet coordinated efforts are in the infancy or, at best, toddler stage of development. In reviewing the comments offered by respondents in this study, we found that only 6 shelters of the 48 responding (8\%) mentioned collaborative arrangements with animal welfare organizations or veterinary clinics to provide temporary shelter for companion animals while a woman resided at a shelter or safehouse. Some respondents said that they allowed companion animals in their shelter (no doubt a challenge, given space and safety issues), others noted arrangements for housing companion animals with a companion animal advocacy program, humane society, animal shelter, and/or veterinary clinic. What are factors that animal welfare organizations need to contemplate to increase such cooperative 
efforts? Are there obstacles to implementing programs to keep companion animals safe while women and children take up temporary residence at shelters?

Let us assume that an animal welfare organization that has facilities for sheltering animals enters into a cooperative agreement with a shelter for women who are battered to board companion animals during a woman's shelter stay. A number of questions arise.

- If animal shelter records are open to public access, could a perpetrator locate his partner by asking to see these records?

- If a companion animal is jointly owned by a perpetrator and his partner, how would the shelter respond to the perpetrator's request (demand?) to claim "his" companion animal?

- Women's stays at shelters are sometimes lengthy - who bears the cost of animal-caretaking during this period?

- If a woman chooses to return home to her partner after a shelter stay and wants to reclaim her companion animal, what steps could be taken to enhance the companion animal's welfare in such a potentially violent environment?

- Perpetrators may at times give their partners companion animals as gifts (e.g., during the forgiveness phase of the cycle of domestic violence). If a companion animal is sought from an animal shelter, could the adopter's background regarding domestic violence be checked?

- Would an animal shelter allow visitations with a boarded companion animal by a woman and her children while they lived away from home? Would this aid or interfere with the companion animal's adjustment to separation?

- If a woman elects to place a companion animal up for adoption because she can no longer care for it or fears for the companion animal's safety if brought home, must she obtain her partner's permission?

- If a companion animal's injuries stemming from abuse necessitate euthanasia, what steps can be taken to minimize the trauma of losing the companion animal for women and children?

- Are shelters equipped to deal with a woman's concern for the welfare of farm animals, who can be targets of abuse in domestic violence situations in rural communities, while she is away from home? 
Effective collaboration between domestic violence services and animal welfare programs will require grappling with issues like these and no doubt other legal and ethical dilemmas arising from attempts to keep women and their companion animals safe.

\section{Implications for Children's Services}

Although the issue of children being traumatized by witnessing domestic violence compounded by witnessing animal abuse warrants more research, we end our discussion by again raising questions about the therapeutic use of animals with child victims of domestic violence and other child-animal relations.

- Animals can help children learn empathy, nonabusive touch, facilitate disclosure about frightening family events, and even be a source of support during court testimony. However, if a symptom of a child's trauma is the child's own abuse of animals, how does one effectively intervene? How would one tailor interventions to children's and adolescent's developmental levels?

- If a child has encountered abusive caretaking and discipline of companion animals by parents, do we know how to counteract such a learning history?

- What are the most effective ways of dealing with the separation, grief, and loss issues for children who have lost contact with their companion animals or seen them destroyed? Both women and children may identify the abuse and killing of companion animals with their own vulnerability.

- Do clinicians see increased phobic behavior toward animals in children, especially in cases where the perpetrator has used an animal as a weapon or sexual "partner" in domestic violence assaults?

- Would understanding a child's history of witnessing and/or engaging in cruelty toward animals facilitate interpretation of the child's responses to projective tests using animal characters (e.g., the Children's Apperception Test, the Blacky Pictures)?

- Are clinicians alert to the possibility that child witnesses of violence and child victims of physical and sexual abuse may display behavioral disorders that include sexual acting out with animals or the use of animals as "instruments" for engaging in self-injurious behavior (e.g., agitating a cat to the point where it scratches the child's limbs)? 
Collaborative approaches between domestic violence and animal welfare programs to intervene in cases of family violence clearly open new horizons in the area of understanding and reducing aggression toward humans and animals. Reaching these common goals remains a challenging and daunting task.

\section{Note}

1. Correspondence should be sent to Frank R. Ascione, Department of Psychology, Utah State University, Logan, UT 84322-2810, or email to FRANKA@fs1.ed.usu.edu. We thank each of the respondents for cooperating in completing our survey and Karen Ranson for her professional services in preparing this manuscript.

\section{References}

Achenbach, T. M., \& Edelbrock, C. S. (1981). Behavioral problems and competencies reported by parents of normal and disturbed children aged four through sixteen. Monographs of the Society for Research in Child Development, 46 (1, Serial No. 188). Achenbach, T. M., Howell, C. T., Quay, H. C., \& Conners, C. K. (1991). National survey of problems and competencies among four to sixteen-year-olds. Monographs of the Society for Research in Child Development, 56 (Serial No. 225).

American Psychiatric Association. (1987). Diagnostic and statistical manual of mental disorders (3rd ed., rev.). Washington: Author.

American Psychiatric Association. (1994). Diagnostic and statistical manual of mental disorders (4th ed.). Washington: Author.

Arkow, P. (1996). The relationship between animal abuse and other forms of family violence. Family Violence \& Sexual Assault Bulletin, 12, 1-2, 29-34.

Ascione, F. R. (in press). Battered women's reports of their partners' and their children's cruelty to animals. Journal of Emotional Abuse.

Ascione, F. R. (1993). Children who are cruel to animals: A review of research and implications for developmental psychopathology. Anthrozoös, 5, 226-247.

Ascione, F. R. (1992). Enhancing children's attitudes about the humane treatment of animals: Generalization to human-directed empathy. Anthrozoös, 5, 176-191.

Ascione, F. R., Thompson, T. M., \& Black, T. (in press). Childhood cruelty to animals: Assessing cruelty dimensions and motivations. Anthrozoös.

Ascione, F. R., \& Weber, C. (1995). Battered partner shelter survey (BPSS). Logan: Utah State University.

Ascione, F. R., Weber, C., Thompson, T. M., \& Wood, D. (in preparation). Pet abuse experiences: Women who are battered and a comparison sample of women without battering experience.

Axtell, J. L. (1968). The educational writings of John Locke. Cambridge: Cambridge University. 
Berrios, G. E. (1996). The history of mental symptoms. Cambridge: Cambridge University. Boat, B. (1995). The relationship between violence to children and violence to animals: An ignored link? Journal of Interpersonal Violence, 10, 4, 229-235.

Browne, A. (1993). Violence against women by male partners: Prevalence, outcomes, and policy implications. American Psychologist, 48, 1077-1087.

Jaffe, P. G., \& Sudermann, M. (1995). Child witnesses of woman abuse: Research and community responses. In S. A. Stith \& M. A. Straus (Eds.), Families in Focus Series, Vol. II. Understanding Partner Violence: Prevalence, causes, consequences, and solutions (pp. 213-222). Minneapolis: National Council on Family Relations.

Jouriles, E. N., Norwood, W. D., McDonald, R., Vincent, J. P., \& Mahoney, A. (1996). Physical violence and other forms of marital aggression: Links with children's behavior problems. Journal of Family Psychology, 10, 2, 223-234.

McNulty, F. (1989). The burning bed. New York: Avon Books.

Munro, H. (1996). Battered companion animals. Irish Veterinary Journal, 49, 712-713.

Osofsky, J. D. (1995). Children who witness domestic violence: The invisible victims. Social Policy Report: Society for Research in Child Development, 9, 3, 1-16.

Pinel, P. (1809). Traité Médico-Philosophique de la Aliénation Mentale (2nd ed.). Paris: Brosson.

Shesgreen, S. (Ed.) (1973). Engravings by Hogarth (Plates 77-79). New York: Dover Publications. 